\section{Case Reports in Dermatology}

\title{
Recurrence of Melanoma after Starting Apremilast for Psoriasis
}

\author{
Thomas G. Salopek \\ Division of Dermatology, Department of Medicine, University of Alberta, \\ Edmonton, $A B$, Canada
}

\section{Keywords}

Melanoma $\cdot$ Psoriasis $\cdot$ Apremilast $\cdot$ Cancer immunosurveillance

\begin{abstract}
This is a report on a 32-year-old man with a history of two previous melanomas with concurrent plaque-type psoriasis. His history dates to 2009, when he was diagnosed with his first melanoma on the right occiput, Clark's level IV, tumor thickness $1.53 \mathrm{~mm}$, nonulcerated, mitotic index $1 / \mathrm{mm}^{2}$. He subsequently developed nodal recurrence after an initial negative sentinel lymph node biopsy and was treated with complete lymph node dissection. In 2012, he was diagnosed with a second primary melanoma on the right upper chest, Clark's level IV, tumor thickness $0.9 \mathrm{~mm}$, nonulcerated, mitotic index $3 / \mathrm{mm}^{2}$. Due to worsening longstanding plaque-type psoriasis in 2015 he was placed on apremilast, with a dramatic improvement in his psoriasis within 4 months of starting therapy. Shortly thereafter the patient developed multiple blue-colored skin papules on the scalp near his first melanoma and on the trunk and upper limbs that on biopsy proved to be due to cutaneous metastasis of melanoma. The patient discontinued the apremilast as there was a concern that his tumor had recurred because of the drug. Apremilast is a phosphodiesterase- 4 inhibitor that impairs the innate immune system, which mediates cancer immunosurveillance. It is postulated that the use of apremilast in our patient resulted in impaired cancer immunosurveillance and led to a recurrence of his melanoma. Although one cannot exclude the possibility of coincidental recurrence of an already metastatic melanoma (to the lymph nodes), caution should be exercised
\end{abstract}


when considering apremilast in the context of patients with known malignancy, in particular melanoma.

\section{Case Report}

This is a report on a 32-year-old man with a history of two previous melanomas with concurrent plaque-type psoriasis. His history dates to June 2009, when he developed his first melanoma on the right occiput, Clark's level IV, tumor thickness $1.53 \mathrm{~mm}$, nonulcerated, mitotic index $1 / \mathrm{mm}^{2}$. He was treated with wide local excision and sentinel lymph node biopsy in June 2009, the latter being negative for nodal metastasis. In November 2009 he presented with a $1-\mathrm{cm}$ blue to black nodule on the right posterior neck, which on complete lymph node biopsy demonstrated nodal metastasis, prompting a complete lymph node dissection in December 2009, during which time 46 lymph nodes were removed, all of which were negative for any additional metastasis. He developed a second primary melanoma on the right upper chest in September 2012, Clark's level IV, tumor thickness $0.9 \mathrm{~mm}$, nonulcerated, mitotic index $3 / \mathrm{mm}^{2}$ that was similarly treated with wide local excision and sentinel lymph node biopsy in November 2012; this too was negative for nodal metastasis.

The patient has a longstanding history of concurrent psoriasis since childhood. He has a strong family history of psoriasis in his sister, father, and maternal grandmother. Despite being of moderate severity, the psoriasis has been largely managed topically with various topical steroids and calcipotriol with betamethasone dipropionate. He had, at one point, a course of acitretin which was ineffective. He never received methotrexate, cyclosporine, or any of the biologics. After being disease-free from his melanoma for more than 3 years, the patient requested a more aggressive therapy for his psoriasis. The safest drug in the context of stable stage IIIB melanoma was thought to be apremilast which he started in August 2015 (30 mg twice a day after initial dose escalation as per drug protocol). At the time of initiation of therapy, he had numerous 2-5-cm plaques on the elbows, knees, thighs, and trunk (PASI 13.1). The patient had a dramatic response to his treatment, and by November 2015 he had just a few scaly pink papules $<1 \mathrm{~cm}$ on the arms, lower limbs, and torso. Within 4 months of starting therapy, the patient had noted a "new" blue 5-mm papule on the left shoulder, which clinically and on dermoscopy was thought to represent a blue nevus. He returned for followup in March 2016 with two new 1-2-mm blue-black papules near the site of his first melanoma on the scalp. Subsequent biopsy confirmed recurrence of his melanoma in the form of satellitosis; molecular testing on this specimen detected the BRAF V600E mutation. He went on to develop multiple additional papules, primarily on the trunk and upper limbs (7 in total), which on biopsy were in keeping with cutaneous metastasis (stage IVa) (Fig. 1). Screening for systemic progression was negative. The patient discontinued apremilast as there was concern that his tumor had recurred because of the drug. He was placed on anti-PD1 therapy with pembrolizumab, which had to be discontinued due to severe liver toxicity and markedly elevated creatine phosphokinase. He is currently being treated with dabrafenib and trametinib. As the patient was started on systemic therapy promptly after being diagnosed with metastatic disease, we cannot comment whether the discontinuation of apremilast resulted in any regression of his cutaneous metastases. 


\section{Discussion}

There is a dearth of information regarding the safety of small molecules (as well as biologics) used in the management of psoriasis in the context of patients with known malignancies. Although apremilast has quickly gained the reputation of being one of the safest drugs for the treatment of psoriasis [1], the exclusion of patients with known malignancies from clinical trials prevents us from stating with absolute confidence that this is in fact true for patients with concomitant malignancies.

Apremilast is a phosphodiesterase-4 inhibitor that results in decreased production of proinflammatory mediators such as tumor necrosis factor alpha, interleukin 23, and interferon gamma, and increased production of anti-inflammatory mediators such as interleukin 10 [2]. Apremilast appears to predominantly effect innate immunity [3]. There is increasing evidence that cancer immunosurveillance is mediated by the innate immune system [4]. Theoretically, drugs that affect the innate immune system may have deleterious effects on cancer immunosurveillance.

It is postulated that the use of apremilast in our patient resulted in impaired cancer immunosurveillance and led to a recurrence of his melanoma. Although one cannot exclude the possibility of coincidental recurrence of an already metastatic melanoma (to the lymph nodes), caution should be exercised when considering apremilast in the context of patients with known malignancy, in particular melanoma, until further work has been done in this area.

\section{Statement of Ethics}

The patient provided consent for publication.

\section{Disclosure Statement}

The author has no conflict of interest to declare.

\section{References}

1 Chimenti MS, Gramiccia T, Saraceno R, Bianchi L, Garofalo V, Buonomo O, Perricone R, Chimenti S, Chiricozzi A: Apremilast for the treatment of psoriasis. Expert Opin Pharmacother 2015;16:2083-2094.

-2 Schafer PH, Day RM: Novel systemic drugs for psoriasis: mechanism of action for apremilast, a specific inhibitor of PDE4. J Am Acad Dermatol 2013;68:1041-1042.

-3 Schafer PH, Parton A, Capone L, Cedzik D, Brady H, Evans JF, Man HW, Muller GW, Stirling DI, Chopra R: Apremilast is a selective PDE4 inhibitor with regulatory effects on innate immunity. Cell Signal 2014; 26:2016-2029.

4 Iannello A, Thompson TW, Ardolino M, Marcus A, Raulet DH: Immunosurveillance and immunotherapy of tumors by innate immune cells. Curr Opin Immunol 2016;38:52-58. 


\section{Case Reports in}
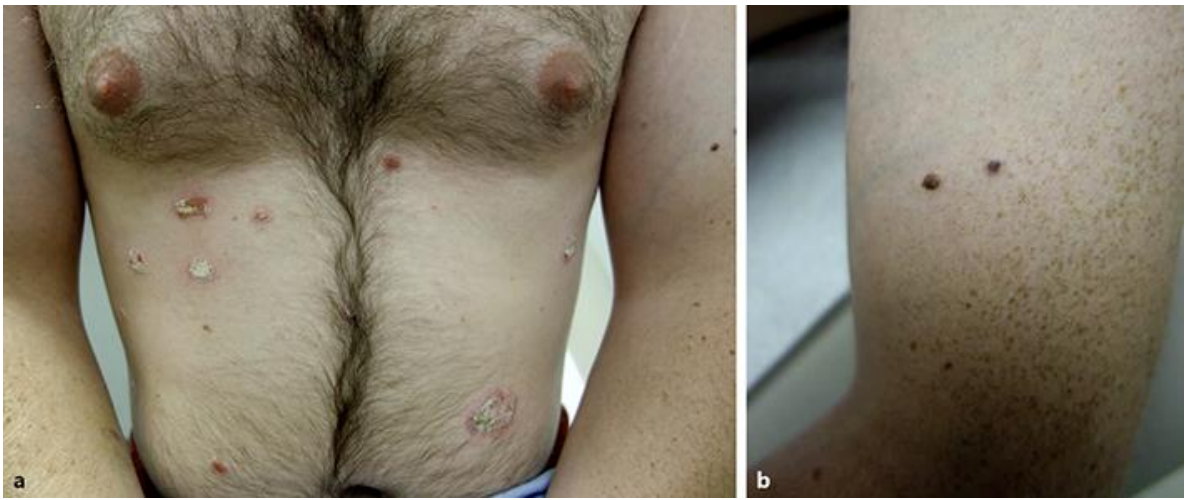

Fig. 1. a Extensive plaques of psoriasis that formed within 2 months of discontinuing apremilast (PASI 12.4). The patient had seven 2-4-mm blue dermal papules on the scalp, upper trunk, and proximal upper limbs as seen on the left arm. b Close-up view of cutaneous metastatic deposit on the left arm. 